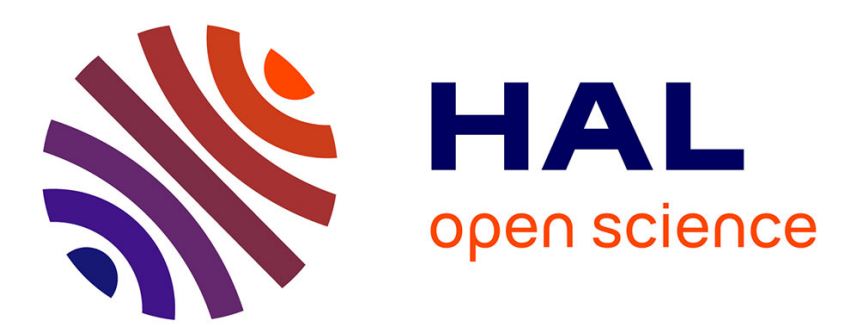

\title{
Mettre en scène le massacre du 24 août 1572 ? La Saint-Barthélemy ou l'actualité théâtrale impossible
}

\author{
Charlotte Bouteille-Meister
}

\section{To cite this version:}

Charlotte Bouteille-Meister. Mettre en scène le massacre du 24 août 1572 ? La Saint-Barthélemy ou l'actualité théâtrale impossible. Littératures classiques, 2012, Ecritures de l'actualité, XVIe -XVIIIe siècle (78), pp.143-164. 10.3917/licla.078.0143 . hal-01406466

\section{HAL Id: hal-01406466 \\ https://hal.parisnanterre.fr/hal-01406466}

Submitted on 5 Dec 2016

HAL is a multi-disciplinary open access archive for the deposit and dissemination of scientific research documents, whether they are published or not. The documents may come from teaching and research institutions in France or abroad, or from public or private research centers.
L'archive ouverte pluridisciplinaire HAL, est destinée au dépôt et à la diffusion de documents scientifiques de niveau recherche, publiés ou non, émanant des établissements d'enseignement et de recherche français ou étrangers, des laboratoires publics ou privés. 


\title{
Charlotte Bouteille-Meister
}

\author{
METTRE EN SCÈNE LE MASSACRE \\ DU 24 AOÛT 1572 ? LA SAINT. \\ BARTHÉLEMY OU L'ACTUALITÉ \\ THÉÂTRALE IMPOSSIBLE
}

Mais [...] qu'est-ce qu'un théâtre « situé »? Et, d'abord, comment un tel théâtre peut-il se positionner, en tant que fiction, par rapport à l'ici et maintenant, par rapport à « l'actualité » vivante ? ${ }^{1}$

Cette interrogation de Jean-Pierre Sarrazac sur la pertinence et l'existence d'un théâtre en rapport direct avec l'actualité nous permet de souligner d'emblée la particularité de l'objet de cet article : la mise en scène de l'actualité sur la scène. De la même façon que l'on reproche a priori au théâtre militant un manque de qualité esthétique et d'ambition formelle, la représentation de l'ici et maintenant sur le théâtre n'a pas bonne presse : la «stratégie du détour ${ }^{2}$ » est presque invariablement valorisée par rapport à la mise en scène du présent selon une vision directe. Pourtant, ce théâtre d'actualité existe à l'époque moderne, en particulier au $X V l^{e}$ siècle et dans le premier tiers du XVII siècle. Dans une thèse soutenue récemment ${ }^{3}$, nous avons défini ce « théâtre d'actualité » selon trois critères : (1) l'univers de représentation mis en scène est en continuité avec l'univers d'existence des spectateurs, quand bien même il serait fictionnalisé par une transposition pastorale ou l'utilisation de personnifications allégoriques ; (2) la fable de la pièce - entendue au sens brechtien - n'est pas tirée d'un ensemble

1 J.-P. Sarrazac, Jeux de rêves et autres détours, Belfort, Circé, 2004, p. 12.

2 ld. , La Parabole ou l'enfance du théâtre, Belfort, Circé, 2002, p. 23.

3 Ch. Bouteille-Meister, Représenter le présent. Formes et fonctions de «l'actualité 》 dans le théâtre d'expression française à l'époque des conflits religieux (1554-1629), thèse de doctorat, Université Paris Ouest Nanterre La Défense, octobre 2011. 
livresque « classique » constituant la culture commune des spectateurs (Bible, mythologie ou histoire passée), mais fondée sur les récits de témoins vivants ou sur des documents historiques de l'histoire en train de se faire, et sur l'expérience d'une situation ou sur le souvenir d'un événement partagés au présent par l'ensemble de la communauté spectatrice; (3) le mode de réception de ces pièces est direct et non pas allégorique (quand bien même la référence serait parfois voilée ou cryptée), c'est-à-dire qu'elles mettent en jeu un ici et un maintenant bien précis, i. e. un événement ou une situation vécus par le public ou du moins proches de lui, et non pas un présent à lire par analogie selon une logique du « détour » entre les lignes d'un passé mythologique ou biblique.

Cette définition posée, nous souhaitons interroger la possibilité de la représentation scénique par ce théâtre d'une actualité éminemment violente : le massacre de la Saint-Barthélemy. Événement hors-norme, dont la cruauté extrême déborde les bourreaux mêmes, la nuit du 24 août 1572 a eu un retentissement national et international extrêmement fort et a donné lieu dans chaque camp à un grand nombre de publications, embarrassées ou virulentes, cherchant à le justifier ou à le condamner. Le théâtre d'actualité du dernier tiers du XVle siècle semble cependant résister à la mise en scène directe de ce massacre, alors même qu'il a mis ou mettra en scène l'actualité violente du massacre des Vaudois en 1545 ou, plus tard, les régicides d'Henri III et d'Henri IV. Est-ce le traumatisme qui impose le silence aux dramaturges protestants ? Est-ce la honte qui conduit les dramaturges catholiques à pratiquer l'art du détour ou du déni dramaturgique ? Est-ce la pudeur, la culpabilité ou les limites d'une esthétique théâtrale fondée davantage sur le discours que sur la mise en scène qui semblent interdire la représentation directe des massacres ? Est-ce en raison de la peur des effets pathétiques que cette représentation pourrait susciter chez les spectateurs et de leur possible utilisation politique en faveur d'une vengeance (public protestant) ou d'une réitération (public catholique) ?

Pour mettre en lumière la spécificité du traitement théâtral de cet événement paroxystique, nous étudierons ici la seule pièce d'actualité en français traitant directement de la Saint-Barthélemy : La Tragédie de feu Gaspard de Coligny ${ }^{4}$, du catholique François de Chantelouve. Pour mieux cerner les enjeux de la mise en

4 Fr. de Chantelouve, La Tragédie de feu Gaspard de Coligny, jadis Amiral de France, contenant ce qui advint à Paris le 24. d'août 1572, avec le nom des personnages, Paris, N. Bonfons, 1575. Les citations renverront à l'édition suivante : La Tragédie de feu Gaspard de Coligny, éd. L. Wolfe et M. Meijer, dans La Tragédie à l'époque d'Henri III, Deuxième série, vol. 1, Florence / Paris, Olschki / Puf, 1999. 
scène de cette actualité traumatique, nous évoquerons ensuite plus rapidement Phaéton, bergerie tragique des guerres et tumultes civiles ${ }^{5}$, pièce de Jean-Baptiste Bellaud, publiée à Lyon en 1574, et qui use d'un détour mythico-pastoral pour proposer une vision catholique du massacre. Cette mise en perspective nous permettra d'interroger la spécificité du théâtre d'actualité catholique face à la représentation de la Saint-Barthélemy, dont l'exceptionnalité violente a d'emblée marqué les esprits de tous bords et de toutes confessions. Nous proposerons enfin en conclusion quelques notes sur The Massacre at Paris ${ }^{6}$ de Christopher Marlowe, pièce jouée au début des années 1590 à Londres et dont les extraits reconstitués qui nous sont parvenus témoignent d'une esthétique protestante du sanglant, radicalement différente de la représentation théâtrale catholique de l'événement.

\section{La Tragédie de feu Gaspard de Coligny : une tragédie d'actualité « sans événement » ou le déni des bourreaux}

La Tragédie de feu Gaspard de Coligny jadis Amiral de France, contenant ce qui advint à Paris le 24. d'août 1572, avec le nom des personnages, écrite par François de Chantelouve, est publiée à Paris en 1575 mais l'autorisation de la Sorbonne, qui se trouve à la fin de la pièce, atteste que la tragédie était achevée avant le 23 octobre 1574. C'est, à notre connaissance, la seule pièce de théâtre d'actualité française à traiter des événements du «24. d'août 1572 », autour desquels elle construit pourtant un silence dramaturgique assourdissant? La Saint-Barthélemy n'est en effet pas seulement - pour reprendre les analyses de Denis Crouzet - un «événement sans histoire », du fait du silence qui s'est immédiatement abattu sur la violence perpétrée cette nuit-là, tant du côté catholique que du côté protestant, enfouissant l'événement « dans l'épaisseur de son passé comme de son futur 》 et donnant lieu à une multiplicité de lectures historiographiques dont nous avons tenté de dégager les grandes orientations ; c'est également une « histoire sans événement », caractérisée par la prolifération des écrits polémiques qui

5 J.-B. Bellaud, Phaéton, bergerie tragique des guerres et tumultes civiles, Lyon, A. de Harsy, 1574. Les citations renverront à cette édition.

6 Chr. Marlowe, The Massacre at Paris: with the death of the Duke of Guise [ca 1592], éd. E. J. Esche, Oxford, Clarendon Press, 1998.

7 Parmi les mises en scène bien plus tardives de l'événement, nous ne citerons que la plus célèbre, le Charles IX ou la Saint-Barthélemy de Marie-Joseph Chénier, intitulée plus tard Charles IX ou l'école des rois, qui dénonce en 1789 l'arbitraire du pouvoir et le fanatisme opprimant la liberté du peuple. 
brouillent les faits et font disparaître le processus de violence « dans les multiples ramifications ou enchaînements des passés ou encore des futurs supposés du massacre de la Saint-Barthélemy ${ }^{8} \gg$.

La Tragédie de feu Gaspard de Coligny est à l'image de la mémoire immédiate des faits qu'elle met en scène : il s'agit d'une tragédie d'actualité événementielle (au sens où elle fait référence à un fait précis et non à une période ou à une situation générale], mais «sans événement ». Tout orientée vers la justification politique du massacre, elle élude complètement la violence qu'il induit et sa représentation. Ce n'est pas seulement qu'elle ne mette en scène ni la tentative d'assassinat contre Coligny ni le massacre du 24 août - ce qui est déjà un élément d'importance dans une tragédie ouvertement inspirée de Sénèque et donc où la figuration d'actes sanglants aurait pu être esthétiquement justifiée - ; ce qui nous interpelle plus particulièrement, c'est la façon dont les tueries sont extrêmement (et c'est un euphémisme) minimisées et surtout dont leur responsabilité est attribuée à un principe providentiel désincarné, qui dédouane les spectateurs de la pièce de leur possible implication dans les massacres.

Le très catholique Chantelouve, chevalier de l'ordre de Saint-Jean de Jérusalem, propose dans sa pièce une justification du massacre du 24 août qui démarque la Lettre à Elvide de Pibrac $^{9}$ et fonde la légitimité de la violence royale sur l'existence d'un complot protestant qui menace la monarchie. En publiant sa tragédie au début de l'année 1575, François de Chantelouve s'inscrit, de plus, dans une double actualité, qui n'est pas sans contradiction. D'une part il s'agit de clamer la légitimité de l'action violente du roi mort et de mettre en garde les protestants contre toute «nouvelle » volonté de rébellion, et ce au moment où le pouvoir peut sembler incertain, voire vacant. II convient d'autre part, en raison du choc provoqué par l'ampleur et la cruauté des tueries de 1572, de clamer l'innocence d'Henri III (Henri d'Anjou au moment de la Saint-Barthélemy) et des Guise, le camp

8 D. Crouzet, La Nuit de la Saint-Barthélemy, Un rêve perdu de la Renaissance, Paris, Fayard, 1994, p. 27, 30 et 99.

9 La Lettre à Elvide (Ornatissimi cujusdam viri, de rebus gallicis, ad Stanislaum Elvidium, epistola, Lyon, B. Rigaud, 1573], rédigée par l'avocat général Guy du Faur de Pibrac, vraisemblablement sur ordre du roi, soulignait dès novembre 1572 que l'action violente du roi à l'encontre des chefs huguenots qui complotaient contre lui était un acte conforme à la justice divine et au droit naturel. La lettre exclut toute responsabilité du souverain et de la famille royale dans les massacres populaires (sans pour autant les condamner explicitement), et l'entière culpabilité de la Saint-Barthélemy est reportée sur l'amiral de Coligny, véritable démon acharné contre la paix et contre le roi. 
des catholiques radicaux plaçant alors tous ses espoirs dans le nouveau souverain qui a succédé à son frère Charles IX et qu'ils croient acquis à leur intransigeance religieuse. Après avoir servi le duc d'Anjou par les armes, quand il commandait les troupes catholiques au siège de La Rochelle au début de l'année 1573, François de Chantelouve met donc sa plume au service de la propagande royale, en rédigeant sa pièce entre mai 1574 , date de la mort de Charles IX, et fin octobre 1574, date de l'autorisation d'impression. Mais si La Tragédie de feu Gaspard de Coligny cherche à légitimer des assassinats politiques ciblés qui ont permis d'éviter un coup d'État, elle ne célèbre en aucune façon un massacre religieux : en 1574, l'« actualité 》 de la Saint-Barthélemy ne semble plus «montrable » dans sa totalité, et nous allons voir comment la pièce de Chantelouve contribue à fixer les limites du souvenir acceptable d'un événement récent, témoignant ainsi en creux d'un phénomène de déni chez les bourreaux catholiques.

\section{La présentation du complot hérétique : discrédit du camp huguenot}

Comme le souligne Olivier Millet ${ }^{10}$, François de Chantelouve utilise un procédé sénéquien qui consiste à conférer au personnage négatif un caractère à la fois infernal (reniant toute croyance religieuse) et tyrannique (échafaudant un complot contre le pouvoir légitime afin d'exercer une puissance sans partage). La pièce s'ouvre ainsi sur un monologue furieux de Coligny, véritable suppôt de Satan, bien plus athée que protestant. Coligny se présente tout d'abord comme « honteux / Qu'un enfant ait bridé [s] on effroyable audace ${ }^{11} \gg$ (v. 2-3). Cet enfant, c'est Henri d'Anjou qui, à dix-huit ans à peine, a remporté avec éclat les batailles de Jarnac et de Moncontour contre les protestants - Chantelouve ne manque pas ici de louer la vaillance militaire du souverain de 1574 , date d'écriture de la pièce. Coligny, humilié et dépité, comme le Satan toujours battu des mystères, invoque ensuite pêle-mêle la mort, la rage, le fer, Pluton, les Furies et Calvin, afin qu'ils lui ouvrent la porte des Enfers où brûlent ses «frères » protestants (v. 7-10). Au Paradis promis aux fidèles catholiques répondent les Enfers mi-chrétiens mi-païens qui attendent les « hérétiques » protestants, le théologien Calvin étant ravalé au rang d'une Furie de tragédie. Le dramaturge discrédite la valeur morale et religieuse

100 . Millet, « L'assassinat politique sur la scène au temps des guerres de religion : trois pièces d'actualité », Vives Lettres, $n^{\circ} 4,1998$, p. 12.

11 Nous avons pris le parti de moderniser systématiquement l'orthographe des textes anciens, à l'exception des cas où les nécessités prosodiques (rythme ou rime) imposaient de la conserver. 
du combat protestant et assimile les hérétiques aux loups dissimulés sous des peaux de brebis qui, dans l'Apocalypse de saint Jean, doivent se répandre dans le monde à l'approche du Jugement dernier. En faisant des personnages protestants des « renard[s] » et des « hypocri[tes] 》 (v. 47-48), Chantelouve relaie l'angoisse eschatologique de la communauté catholique qui voit la présence des hérétiques dans le monde comme un signe indubitable de sa fin imminente. L'auteur apporte ainsi la première justification du massacre de la Saint-Barthélemy, qui est celle de toute guerre sainte : l'assurance du salut pour les bourreaux.

Pour parachever cette présentation négative, Chantelouve fait de son personnage faible et lâche un désespéré, c'est-à-dire un pécheur aux yeux de l'Église. Après avoir voulu se tuer au début de son monologue, Coligny se plaint à Fortune que tous ses efforts guerriers ont été réduits à néant par « un Pyrrhe nouveau » (Henri d'Anjou, à nouveau loué pour sa valeur militaire). II décide donc de « fiche [r] tout [son] espoir sur la désespérance » (v. 100) et énonce clairement son projet :

Je veux doncques mourir, ou bien tuant le Roi

Transférer cautement la couronne sur moi. (v. 101-102)

Coligny, démon hérétique désespéré, est ainsi présenté comme un traître à la fois envers son roi contre lequel il complote, envers sa religion qui n'est qu'un masque, et envers ses alliés qui pensent qu'il se bat pour défendre la religion réformée. Au regard de la fidélité que Coligny témoigna à Charles IX et que tous les historiens attestent, Chantelouve se livre ici à une falsification historique manifeste, visant à désigner à l'exécration du spectateur le Mal, l'adversaire absolu. Au niveau tant dramaturgique que politique, le portrait négatif de Coligny permet de justifier les vengeances divine et royale qui vont suivre, légitimées par cette démonstration d'hérésie comme par le complot échafaudé contre le roi.

À la scène 2 de l'acte I, le camp du Mal est renforcé par l'entrée en scène de Gabriel de Lorges, comte de Montgomery, l'un des chefs du parti protestant depuis son retour d'Angleterre en 1562. Le choix de ce personnage n'est pas fortuit : il permet d'étayer la thèse d'un complot protestant persistant contre la monarchie des Valois. Montgomery rappelle en effet qu'il est responsable de la mort d'Henri II, mais de telle façon que l'éclat de sa lance qui blessa mortellement mais accidentellement le Roi semble avoir été un coup intentionnel : «Et tout ainsi que de mon bras jadis / Je renversai mort le Roi à Paris » (v. 127-128). Le fait qu'en 1559, date de la mort d'Henri II, ni Coligny ni Montgomery ne se soient encore convertis au protestantisme n'arrête en rien Chantelouve, ni l'ensemble de la propagande catholique. Montgomery affirme ensuite vouloir « tuer le Roi, et massacrer son 
frère », parachevant l'entreprise commencée avec leur père et mettant l'ensemble de la monarchie Valois en péril, y compris le roi actuel de 1574 .

\section{Le roi défunt seul responsable, ou comment épargner les vivants}

Le début de l'acte II se déroule au Louvre, après l'entrée en vigueur de l'Édit de Saint-Germain, mais avant la participation de Coligny au Conseil du Roi, soit entre août 1570 et septembre 1571. Si l'acte I est l'acte de Coligny, l'acte II est l'acte du Roi : la présentation en diptyque est parfaitement symétrique car au monologue de l'amiral répond à l'ouverture de l'acte suivant le monologue de Charles IX, à peine défunt au moment de l'écriture de la pièce. Le roi qui se présente au spectateur n'est pas un roi en majesté : il se dit en effet « chétif » et « misérable 》 (v. 341), d'une part parce que ses sujets se sont rebellés contre lui alors qu'il était encore « pendant à la mamelle » (v. 343) - allusion à la conjuration d'Amboise de 1560, et d'autre part par ce que son « peuple gaulois / Endure tant de maux » dans les guerres civiles qui ravagent le pays (v. 346). La figure royale est cependant complexe : en tant que chef de royaume, le Roi connaît les volontés mutines de ses sujets rebelles ( « Ma ruine, ma fin, mon royaume, mon sang, / Est l'aiguillon seulet qui vous pique le flanc », v. 381-382) ; en tant que fils aîné de l'Église catholique, il est clément et doux et redoute de verser le sang de ses sujets («Vu que je suis contraint couper ma propre chair ? », v. 374) ; enfin, en tant que premier seigneur du royaume, c'est un roi combattant qui sait convoquer dans son discours les grandes victoires catholiques - lesquelles sont cependant l'œuvre de son frère et qui annonce qu'il est prêt à se sacrifier, s'il est sûr d'assurer ainsi la paix du royaume.

Ce monologue, qui n'a de délibératif que la forme, est bien davantage un second monologue d'exposition et ne permet pas au Roi de prendre une décision. II décide donc de demander l'avis de son Conseil pour savoir s'il doit faire la paix ou continuer une guerre qui lui pèse. Dans l'hypothèse où les rebelles se dresseraient contre le Roi, voici l'attitude que le Conseil préconise : «tuez, meurdrissez, fracassez / Et les Géants sous Etne renversez » (v. 469-470). Bien que la référence topique au combat des Géants contre les dieux de l'Olympe l'euphémise quelque peu, la violence de cette imprécation semble contradictoire avec les souhaits de paix exprimés précédemment dans la scène par le Conseil. Est-ce pour Chantelouve une façon de montrer que ce «règne de l'amour » est un « rêve perdu » (Denis Crouzet) auquel un soldat catholique militant ne peut souscrire ? De cette façon, l'auteur peut signifier que le Roi ne sera pas entièrement pris au dépourvu par la trahison huguenote, et résoudre ainsi la difficulté qui consiste à mettre en scène une 
figure royale à la fois catholique (qui pardonne), politique (qui n'est pas dupe) et majestueuse (qui n'est pas machiavélique). Chantelouve est ici fidèle au récit de Pibrac, selon lequel le roi et les princes de sang étaient partisans de la clémence, mais ont dû céder à la raison de plusieurs «vieillards personnages de très grande expérience ${ }^{12} \gg$ selon lesquels il était nécessaire de décapiter le complot. Cette évocation précise et virulente de la violence, qui anticipe sa justification, permet enfin au dramaturge de préparer le spectateur au massacre de la Saint-Barthélemy, qui n'aura pas lieu sur scène et dont l'auteur choisit de ne convoquer les souvenirs sanglants que par des mots.

Le Chœur clôt l'acte en «salu[ant] la paix »: par la mise en scène d'un Peuple français désireux de « repos » (v. 576), le dramaturge cherche à dédouaner le peuple parisien de sa participation aux agitations qui précédèrent le massacre de la Saint-Barthélemy, et à attribuer l'entière responsabilité des troubles au camp protestant ainsi qu'à une volonté divine supérieure qui, sous la forme d'un Jupiter tonnans, va venir troubler cette pseudo-conclusion irénique, transformant un massacre très politique en catastrophe « naturelle », ininterprétable en termes humains.

\section{La lisibilité scénique de l'opposition axiologique}

Le troisième acte s'ouvre sur un monologue de l'« angeliqu' Mercure » (v. 602) qui descend des cintres pour annoncer au spectateur qu'il s'est acquitté de la charge que lui a confié son père Jupiter : il a « meurtri la meurtrière main » de l'amiral et fait « Du sang gaspardin un flot large, / [...] bouillonner soudain 》 (v. 722-724). On aura reconnu l'attentat du 22 août 1572 contre Coligny. Les responsabilités humaines sont cependant gommées : c'est Jupiter-Dieu qui frappe l'amiral et non un sbire à la solde des Guise ou de l'Espagne ; l'attentat apparaît comme une punition divine des péchés de Coligny et non comme une manœuvre du camp ultra-catholique. Le monologue de Mercure-Gabriel vient trancher l'opposition entre les deux camps et permet de s'assurer que le spectateur a choisi le Bien contre le Mal, tout en soulignant l'efficacité du messager de la Providence, qui décide du cours des actions humaines et maîtrise toutes les ficelles de l'intrigue : politiques, historiques, mais aussi théâtrales. C'est lui qui annonce l'entrée en scène de Montgomery, «Polyphème Lorges » (v. 729), et du capitaine Piles qui réitèrent leur volonté de tuer le Roi le lendemain, étayant une fois encore la thèse du complot protestant.

12 Cité dans D. Crouzet, op. cit., p. 102. 
La menace infernale des hérétiques se fait de plus en plus présente sur la scène et pressante dans l'action à l'acte IV. Son ouverture est ainsi hautement spectaculaire et use de l'effet d'horreur pour confirmer le spectateur dans sa haine du protestant. Suivant un effet de symétrie par rapport à l'acte III, François de Coligny d'Andelot, frère de l'amiral mort en 1569, surgit sur la scène par les dessous du théâtre. Tout comme Mercure était chargé d'une mission divine, d'Andelot a été envoyé par l'Enfer pour exciter Coligny à la vengeance contre le Roi et contre les Guise qui soutiennent l'Église catholique. L'effet spectaculaire est encore renforcé par l'apparition des Furies qui viennent exhorter le camp protestant à la révolte et à la guerre, avant de retourner avec d'Andelot « aux abîmes ombreux » (v. 1008). Coligny, seul en scène, jure alors de tuer Guise et le Roi.

Si elle n'apporte pas d'élément nouveau pour l'action, l'apparition infernale d'Andelot renforce le sentiment d'urgence et confirme le lien que trace scéniquement Chantelouve entre protestantisme et hérésie. Le parallèle entre l'apparition de Mercure et celle du frère de Coligny permet au dramaturge de souligner de manière manichéenne la séparation axiologique entre les deux camps, étant bien entendu qu'il n'est pas question d'évoquer ici la séduction du Mal. Le théâtre d'actualité catholique suscite les images spectaculaires et terrifiantes des Enfers pour stigmatiser son ennemi religieux dont il peut ainsi montrer l'inévitable damnation : par une transcription scénique de la «pastorale de la peur ${ }^{13}$ » que pratiquent les prêcheurs et en particulier les curés parisiens, l'ombre d'Andelot et les repoussantes Furies servent tout autant à l'édification du public qu'à la justification du massacre voulu par Dieu et souhaité par le Peuple français après cette démonstration du caractère infernal des protestants :

Seigneur retiens la bride à l'amiral hautain,

À notre prince étends le bras humain. (v. 1035-1036)

\section{La victoire d'une providence divine et royale}

La décision divine doit cependant encore trouver une forme politique et humaine pour s'accomplir dans le monde et c'est le rôle de la première scène de l'acte V. La scène se déroule au soir du 23 août ou au petit matin du 24 août : après un dialogue en stichomythie qui oppose le souverain défendant le « règne de

13 J. Delumeau, Le Péché et la peur. La culpabilisation en Occident (XIII -XVIII ${ }^{e}$ siècles), Paris, Fayard, 1983, p. 358. 
l'amour » et son Conseil l'incitant à exécuter les comploteurs, l'ordre du massacre est finalement arraché au Roi. Dans la scène suivante, le Messager annonce déjà au Peuple français la mort (hors-scène) de Coligny et des autres chefs protestants. En plaçant l'exécution entre la première et la deuxième scène du dernier acte, et non entre les deux derniers actes, Chantelouve évite qu'elle soit l'acmé dramatique de la pièce et désamorce une possible quoique très improbable déploration des morts de la Saint-Barthélemy, en choisissant pour « catastrophe » à sa tragédie non les tueries mais la rébellion contre le Roi. Quand le Peuple, en position de coryphée, demande au Messager quelles sont les « nouvelles 》 (v. 1115), ce dernier lui annonce qu'elles sont « très cruelles 》 (v. 1116) : « L'amiral et les siens d'une traîtresse audace / A conspiré la mort de la royale race » (v. 1119-1120). À nouveau, le Messager légitime l'acte royal par la théorie du complot et évoque l'exécution des comploteurs en seulement quatre vers :

En gagnant le devant [sur le complot], il [le Roi] envoya grand' force

Tandis que tout Paris ès cantons se renforce,

Qui Piles, l'amiral, Pardaillan, et Pinos,

Et autres envoya sous les stygiens flots. (v. 1135-1138)

Le massacre n'a pas de «massacreur » grammaticalement stable : le sujet du verbe « envoya » est-il l'impersonnelle « grand' force » envoyée par le Roi ou bien le très général « Paris » ? L'ambiguïté n'est pas anodine eu égard au débordement de violence dans les foules parisiennes une fois les ordres du souverain exécutés. L'antéposition de «Piles, l'amiral, Pardaillan et Pinos » tend de plus à transformer la phrase en structure passive qui fait disparaître l'agent de mort dans les silences de la syntaxe et de la scène, quand le recours au détour mythologique (les « stygiens flots 》) permet à la fois de rappeler le caractère infernal des huguenots et d'effacer le souvenir trop (?) concret des eaux rouges de la Seine.

Le Chœur décrit ensuite le destin de Coligny - sans jamais le nommer - comme un exemplum moral et didactique du sort de l'ambitieux : celui qui désirait régner sur la France possède le «plus haut lieu qui soit en Montfaucon » (v. 1188). Le traitement dégradant que l'on fit subir à la dépouille de l'amiral est ainsi justifié par la morale de l'aurea mediocritas du Peuple français qui cherche à convaincre le public du bien-fondé de ce dépeçage post mortem, sans pour autant mentionner sa participation au supplice.

«Ce qui advint à Paris le 24. d'août 1572 », dans la représentation qu'en donne François de Chantelouve, c'est donc la rébellion d'un petit groupe d'individus que Dieu, par le bras armé du souverain légitime et de son conseil, a punis de leur impiété. La « tragédie » de Gaspard de Coligny n'est pas la mise en scène d’un crime 
d'État, mais la représentation de la punition d'un hybris intolérable. En achevant l'action dramatique le 24 août et en évoquant de manière plus précise la seule mort de Coligny, le dramaturge rejette dans l'ombre de la scène les massacres à grande échelle perpétrés à Paris et en province, massacres qui ne pourraient plus être justifiés par la théorie du complot. En cantonnant le Peuple français à un rôle d'observateur extérieur à l'action divine et royale, qui apprend toujours après coup les décisions et les actions des personnages, Chantelouve l'innocente dramaturgiquement de sa participation réelle aux tueries en effaçant la dimension collective et hystérique du massacre - « un massacre sans massacreur ${ }^{14}$ » ? Mais pourquoi ce silence dans un texte dont on ne peut douter du caractère catholique militant?

\section{Trauma et déni : les raisons d'un silence scénique}

Dans La Tragédie de feu Gaspard de Coligny, il s'agit pour Chantelouve non seulement de ne pas montrer, mais également de taire et de dissimuler les massacres d'août 1572. Or ce silence dit, en creux, quelque chose de la manière dont les catholiques ont réagi à la Saint-Barthélemy. Le silence de Chantelouve pourrait trouver une explication esthético-politique, si l'on admet que la mise en scène des massacres (comme de la tentative d'assassinat sur la personne de l'amiral) courait le risque d'éveiller la pitié et la compassion du public envers des personnages dont le dramaturge souhaite qu'ils demeurent jusqu'au bout des rebelles infernaux. Cette hypothèse ne tient cependant pas tout à fait si on analyse la façon dont les scènes de bataille fonctionnent dans certaines pièces de Shakespeare, où la représentation scénique de la destruction sanglante de l'adversaire est présentée comme une chasse joyeuse contre des ennemis bestialisés.

II n'en reste pas moins que construire une tragédie d'actualité sur un événement pour le nier dramaturgiquement est une pratique d'écriture étonnante. Nous sommes loin ici des médailles à la gloire du massacre frappées par la papauté et l'on ne peut que constater l'absence de triomphalisme dont fait preuve l'auteur catholique, et avec lui l'ensemble de la production française justifiant pourtant la réaction violente du souverain à l'encontre des huguenots. Nul texte d'aucune sorte ne glorifie les massacres de masse, ce qui marque la gêne de l'opinion catholique face à des événements qu'elle a pourtant suscités et auxquels elle a, pour une large part, participé. Après les tueries frénétiques de la fin de l'été 1572,

14 D. Crouzet, op. cit., p. 100. 
les catholiques semblent se réveiller d'une hystérie collective et se trouver dans l'impossibilité de prendre en charge les violences extrêmes commises à l'encontre des protestants. Le traumatisme provoqué par le spectacle des meurtres, des tortures, des profanations de cadavres, paraît toucher l'ensemble de la population qui en a été témoin, les victimes comme les bourreaux, les protestants comme les catholiques:

L'évènement [la Saint-Barthélemy] est différence, une telle différence par rapport au long fil de l'histoire humaine, qu'il laisse ceux qui l'apprennent dans une situation de déroute devant toute explication rationnelle, devant toute faculté de narration. ${ }^{15}$

La manière dont François de Chantelouve met en scène par l'absence la nuit de la Saint-Barthélemy relève cependant également de sa stratégie de propagande. En centrant l'action sur les seuls personnages de l'amiral et de ses proches, en en faisant un ambitieux qui menace l'autorité royale et qui est puni (avant tout) par la providence divine et (un peu) par le Conseil anonyme d'un roi défunt, le dramaturge cherche à mettre en avant une culpabilité politique du camp protestant. Cette culpabilité, qui, à aucun moment de la pièce, ne ressortit à la foi ni à une préoccupation eschatologique, puisque le protestantisme est assimilé à un athéisme infernal, appelle un châtiment qui ne peut plus, dès lors, paraître tyrannique. Chantelouve souhaite également taire les responsabilités du souverain vivant (Henri III), de l'entourage royal (Catherine de Médicis, les Guise), et de l'ensemble du «Peuple français » dans les violences, et en cela il agit en fidèle serviteur de la cause catholique. À l'été 1574 la couronne de France n'est pas en effet consolidée et le pouvoir d'Henri III a besoin de s'affirmer, face aux protestants mais également face à ses propres sujets catholiques. En transformant les massacres d'août 1572 en tragédie d'un complot avorté et légitimement combattu par un roi en danger, La Tragédie de feu Gaspard de Coligny sert ce double projet de propagande : légitimer la mise à mort des chefs protestants rebelles et exclure génériquement de la scène d'actualité un «Peuple » toujours difficile à contrôler. Le choix d'une dramaturgie tragique antiquisante ${ }^{16}$ participe en effet du silence de la pièce sur les actions violentes perpétrées par le peuple : la tragédie d'inspiration sénéquienne, si elle n'exclut nullement la représentation du sang versé et des cadavres, ne comprend pas, traditionnellement, de scènes de groupe ou de guerre.

15 Ibid., p. 34.

16 Ce choix est particulièrement sensible dans la répartition en cinq actes, la présence systématique du chœur à la fin de chaque acte et la part importante des monologues (notamment le monologue protatique). 
Le choix de cette influence antique semble être déterminant dans la stratégie de Chantelouve : il permet à la fois la représentation d'une hybris monstrueuse et punie (Coligny), tout en dédouanant les Parisiens de leur participation au massacre, puisqu'ils ne peuvent être représentés sur la scène que sous la forme d'un chœur passif.

Le trou dramaturgique que le dramaturge crée dans La Tragédie de feu Gaspard de Coligny relève ainsi sans doute autant du trauma que du déni. Les dramaturges réformés semblent avoir besoin d'un temps long entre l'assassinat de leurs coreligionnaires et sa représentation, comme le montre le laps de temps écoulé entre le massacre des Vaudois de 1545 et l'anonyme Tragédie du Sac de Cabrières $^{17}$ (ca 1566) ou entre la Saint-Barthélemy et The Massacre at Paris de Christopher Marlowe ( ca 1592). Cet éloignement témoigne de la nécessité d'un sas temporel au cours duquel la mémoire protestante peut apprivoiser les événements traumatiques pour pouvoir, éventuellement, les représenter à travers une fiction scénique qui ne passe pas par le détour allégorique. Pour les dramaturges catholiques, au contraire, le temps qui passe les éloigne de l'enthousiasme suscité par la « saison des Saint-Barthélemy » et le triomphalisme catholique de l'automne 1572 laisse place à un silence gêné, voire à un déni des massacres incontrôlés. La distance par rapport à l'événement (même de deux ans seulement) rend sa représentation sur la scène d'actualité catholique problématique : s'il est nécessaire d'évoquer la nuit du 24 août pour la justifier et pour légitimer un nouveau roi qui a été très impliqué dans les massacres, il convient pour les auteurs de jeter un voile dramaturgique sur les massacres collectifs et les violences extrêmes, ou bien de passer par le détour mythologique, comme le fait Jean-Baptiste Bellaud dans son Phaéton.

\section{Contrepoint et détour : le recours à la mythologie dans le Phaéton de Jean-Baptiste Bellaud}

En contrepoint de notre étude de La Tragédie de feu Gaspard de Coligny, nous souhaitons à présent évoquer plus rapidement une pièce qui n'appartient pas au théâtre d'actualité tel que nous l'avons défini en introduction, et ce afin de souligner la spécificité de l'événement de la Saint-Barthélemy et les problèmes de représentation qu'il soulève pour les dramaturges catholiques qui souhaitent

17 Tragédie du sac de Cabrières, ms., Bibliothèque Vaticane, Codex pal. lat. 1983. On pourra se reporter à l'édition suivante : Tragédie du sac de Cabrières, éd. D. Bocassini, dans La Tragédie à l'époque d'Henri Il et de Charles IX, Première série, vol. 3, Florence / Paris, Olschki / Puf, 1990. 
le mettre en scène. Phaéton, bergerie tragique des guerres et tumultes civiles est publié en 1574 à Lyon par un certain Jean-Baptiste Bellaud, originaire de l'Anjou si l'on en croit les dédicaces qui ouvrent le volume, et dont les obédiences catholiques, apostoliques et romaines ne font aucun doute. L'intrigue de sa « bergerie tragique » reprend le mythe de Phaéton tel qu'il est exposé par Ovide au livre II des Métamorphoses, en y mêlant des personnages empruntés à d'autres épisodes des Métamorphoses et des Fastes, mais également aux Bucoliques de Virgile. À l'acte I le berger Orphée annonce les incendies que s'apprête à allumer Phaéton et qui vont dévaster le monde et plus particulièrement le royaume de France :

Le seul Loire, la Seine, et les flots écumeux,

Du Rhône, et de Durance, oncques n'avaient tels feux

Ressenti fièrement, ni le Tar [sic], ni Garonne,

La Saône, Meuse, et Marne, et Charente, et Dordonne,

N'ayant jusques ici reconnu dans leurs bords

De monstres si hideux et flamboyants efforts. (p. 1)

L'ombre d'Eurydice, évoquée par Orphée, prédit la fin de Phaéton et la victoire de « ce brave berger qui jeune s'achemine, / Par mille et mille feux où le ciel le destine $\gg($ p. 6).

L'acte II met en scène Épaphus (roi d'Égypte et personnage du mythe de Phaéton], qui a été ligoté par Clyméné, la mère de Phaéton, et qui déplore la situation de la maison de Phœbus, où « une Églé, sans troupeau ni pasteur, / Errante et soupirant presque tout' éperdue / Se verra méprisée comme vieille et chenue » et dans laquelle les brebis « délaissent les herbis, / Et parcs accoutumés à prendre leur pâture, / Pour s'éblouir des rais qui changent d'heure à heure » (p. 8). Cette Églé, « épouse de Phœbus », n'est pas facilement identifiable, et son nom semble avoir été choisi principalement en raison de sa proximité phonétique avec le mot Église. Marsyas et Ocyrroé entrent en scène ; Épaphus les prie de le délivrer. Marsyas - il porte le nom du satyre qui ayant défié les qualités de musicien d'Apollon-Phœbus fut soumis au supplice de l'écorchement pour punir son hybris - refuse, car il a « quitté d'Eglé le pompeux avantage » (p. 9). Ocyrroé (fille de Chiron transformée en jument par la colère de Jupiter) refuse quant à elle pour venger sa famille, dont les biens ont été confisqués par Épaphus. Protée (divinité marine, donc opposée au feu que répand Phaéton) se lamente de l'état dans lequel Clyméné a mis le parc français ; il rencontre Épaphus et lui conseille de promettre la liberté à Marsyas et Ocyrroé pour obtenir la paix. Épaphus accepte pour « soulager [s] es troupeaux périssant » (p. 14) et le Chœur se réjouit d'un bonheur retrouvé pour la France. 
La vision d'Orphée et Eurydice se réalise à l'acte III : Phaéton se plaint qu'on ne le laisse pas exercer la charge de son père, et qu'on le considère comme un enfant illégitime, Phœbus préférant «l'étranger » (p. 17). Méris (nom de l'un des deux interlocuteurs dans la neuvième des Bucoliques de Virgile, qui représente un berger chassé de ses terres par les guerres civiles] tente de le calmer en lui disant qu'il est fils d'Églé et de Phœbus, qui a pris « des mortels la nature » pour rappeler les troupeaux « dans ses parcs » et leur a choisi Églé comme seule «nourrice » (p. 19). Phaéton refuse cependant de ne pas venger l'affront qui lui est fait et désire éclairer le monde. Le Chœur blâme son ambition et appelle la colère de Dieu sur les puissants qui imposent leurs maux sur le peuple.

Au début de l'acte IV, Eglé implore Pan de ne pas laisser périr ses troupeaux entre les mains de Phaéton qui les ravage par la « flamme civile 》 (p. 24), car il se révèle incapable de conduire le char de Phœbus. Elle appelle à l'aide Méris et Aristée (berger ou divinité champêtre, caractérisé dans la mythologie par sa fidélité aux dieux) ; les deux bergers décident d'aller trouver Épaphus pour le pousser à l'action radicale contre le fauteur de troubles qui «s'élance finement contre la royauté » (p. 29). Épaphus se résout alors à envoyer ses troupes contre Phaéton :

Sus fidèles pasteurs, jà le ciel vous appelle,

Pour revancher les parcs amortissant les feuz

Par une Clyméné cruellement conceuz. (p. 28)

Phaéton et Marsyas meurent, Aristée et Méris se réjouissent en proclamant la victoire d'Épaphus et le Chœur implore le Ciel de détourner le malheur « de nos cités 》, qui ont «irrité / Les vertus éternelles / Par nos guerres cruelles » (p. 33).

L'acte $V$ est l'acte de déploration des Héliades, les sœurs de Phaéton. L'une d'entre elles décrit un royaume ravagé puis annonce à Clyméné la mort de son fils, en « lui montr[ant] le corps à terre » (didascalie, p. 35) : « Voilà le corps en terre, / Froissé de mille coups par ce brillant tonnerre ». Clyméné pleure son fils, dont elle emporte la dépouille en fuyant Épaphus, qui entre en scène pour regretter le saccage de ses parcs et la mort de ses agneaux. II se résout cependant à « hasarder [...] un effort horrible » contre ses ennemis rebelles, afin de «leur montrer les effets d'un pouvoir invincible » (p. 37-38). Aristée conclut la «bergerie tragique » en espérant que Pan, par la mort de Phaéton, « enseveli[sse] l'envie ».

Dans ce patchwork mythologico-littéraire, il est plus ou moins aisé de reconnaître à quels personnages historiques réels renvoient les différentes figures : sous l'œil de Pan-Dieu et de son fils Phœbus-Christ, le roi Épaphus est Charles IX, fidèle défenseur d'Églé (I'Église catholique), désireux d'épargner ses 
sujets, mais qui se résigne in fine à l'action violente contre les rebelles. II est aidé dans sa lutte par Méris et Aristée, qui désignent vraisemblablement le duc et le cardinal de Guise. Le personnage de Protée, qui conseille au roi la paix avec les rebelles protestants (l'allusion à la paix de Saint-Germain est transparente) est plus difficile à identifier : il s'agit très certainement d'un « politique » (i.e. catholique modéré fidèle au roi], que sa souplesse en matière religieuse pourrait rapprocher des métamorphoses de la divinité marine; on peut alors avancer le nom de Michel de L'Hospital, mort en 1573 à 68 ans, et que pourrait donc représenter sur la scène le « vieillard de la mer » Protée - même s'il ne prit pas part aux négociations de 1570. Le camp des rebelles à l'autorité divine et royale est mené par PhaétonColigny, présenté comme un ambitieux qui veut avant toute chose conquérir le pouvoir ; il est fils de Clyméné (l'hérésie protestante) et soutenu par Marsyas (peut-être Montgomery, si l'on admet que la pièce a été écrite après juin 1574, date de son exécution] et Ocyrroé, que nous n'avons pu relier à une personnalité protestante historique, mais qui devait être identifiable pour les contemporains, sans doute grâce à la métamorphose en jument qui pourrait renvoyer à un blason ou au nom d'un domaine.

La particularité de la pièce de Jean-Baptiste Bellaud réside dans la conjonction de l'usage d'une structure narrative préexistante (le mythe de Phaéton qui a voulu conduire le char du Soleil) et d'une lecture «à clefs », qui implique que le spectateur établisse des rapprochements constants entre les personnages de la fiction et la réalité présente (ou à peine passée) à l'extérieur de la séance théâtrale. La structure du mythe (doute sur l'ascendance de Phaéton, catastrophe de sa rébellion qui conduit à sa mise à mort, déploration de sa mère et ses sœurs] est représentée, mais elle est également profondément modifiée par l'intervention de personnages extérieurs au mythe, qui doivent être identifiés par le public comme des personnages historiques pour que la fable prenne véritablement sens. De plus, une distorsion très grande est apportée au récit ovidien : ce n'est pas Zeus qui foudroie Phaéton, mais les armées humaines du roi Épaphus.

Phaéton, bergerie tragique est ainsi un cas-limite entre le théâtre qui utilise le détour mythologique ou biblique pour figurer, par allusion seulement, la réalité des guerres civiles, tout en respectant l'intégrité de l'épisode convoqué sur la scène, et le théâtre d'actualité qui met en scène les événements récents selon une structure dramaturgique conforme au réel (ou du moins à ce que l'on voudrait qu'il fût), en utilisant parfois des personnages allégoriques ou en cryptant les noms des personnages. Le mythe de Phaéton est détour détourné car le réel qu'il pourrait simplement évoquer selon le modèle d'un exemplum moral (la punition de l'ambition par la force légitime) qu'il appartiendrait au spectateur de relier 
(ou non) à son présent, sans que cela influe sur la fable mise en scène, vient au contraire ici en informer la composition.

On peut cependant s'interroger sur la fonction de ce détour détourné. S'il s'agit pour Jean-Baptiste Bellaud de mettre en scène une allégorie aussi transparente des « guerres et tumultes civiles », pourquoi ne choisit-il pas le théâtre d'actualité ? Qu'est-ce que le recours au mythe de Phaéton, réécrit pour les besoins de la cause allégorique et apostolique, permet de mettre en scène ? Précisément, le massacre de la Saint-Barthélemy, à travers la dépouille consumée de l'orgueilleux qui a voulu se mesurer au Soleil. L'insistance sur le démembrement du corps de Phaéton dans la déploration de Clyméné est doublement saisissante. D'une part parce qu'elle n'imite en rien la description d'Ovide, qui n'évoquait que rapidement le « cadavre consumé par la flamme aux trois dards ${ }^{18} \gg$; d'autre part parce qu'elle correspond en tous points aux sévices qui furent infligés au cadavre de Coligny le 24 août 1572 :

las! tes mains courageuses

Pour être à tes haineux d'un laurier odieuses, Ne sont plus à tes bras : la jambe te défaut, Pour avoir cheminé d'un cœur si grand et haut, Qu'on peut pour un jamais en remarquer la trace?

Las! je vois de ton sang rougissante la place, Honteuse de te voir sans les membres honteux:

Tournant même ton corps et ton cœur vers les cieux, Pour de tes ans passés témoigner la constance Qui donc pourrait souffrir si grande violence ? (p. 35, nous soulignons)

Le corps du Phaéton de Bellaud, vraisemblablement représenté sur la scène par une feinte, n’a rien de la dépouille frappée par le «brillant tonnerre » (p. 35) de Jupiter : elle est sanglante, dépecée, privée de ses mains, d'une jambe et de ses organes sexuels, comme le cadavre de l'amiral que l'on a pendu à Montfaucon. Cependant ce corps est double : il est tout de même, dans la fiction, celui de Phaéton et, seulement au niveau allégorique, celui de Coligny. Cette double lecture toujours maintenue crée certainement la distance nécessaire à la représentation sur la scène de l'assassinat de l'amiral, scandale d'État et paroxysme de violence, qui pourrait susciter la pitié du spectateur plus sûrement que le sort si lointain et fictionnel de l'ambitieux Phaéton.

La violence n'est cependant pas limitée au châtiment du fils de Phœbus dans la pièce de Bellaud. Sans qu'aucune autre mort que celles de Phaéton et Marsyas

18 Ovide, Métamorphoses, II, v. 325-326 : « trifida fumantia flamma / Corpora ». 
ne soit mentionnée ou nécessitée par la fable, l'une des Héliades fait surgir la vision de nombreux corps morts dont coulent « mille ruisseaux » de sang: « Je ne puis nulle part fuyante m'en aller, / Sans voir de ces corps morts mille ruisseaux couler » (p. 33). De même, alors que la pièce s'est concentrée sur la révolte presque individuelle de Phaéton contre l'autorité d'Épaphus, le roi décide à la fin de la pièce un effort militaire violent contre d'autres sujets rebelles. Le spectateur de 1574 doit nécessairement entendre, dans ces violences qui n'appartiennent ni au mythe ni même vraiment à la fable représentée, l'écho des tueries massives de la SaintBarthélemy et des ruisseaux de sang qui ont rougi Paris et de nombreuses autres villes du royaume. Ces évocations sont cependant relativement subliminales dans l'économie de la fiction, et l'on constate à nouveau la difficulté d'un dramaturge catholique à dire les massacres populaires, qui sont repoussés dans l'au-delà de la pièce et cautionnés par l'autorité royale de manière anticipée.

Le détour par le mythe semble donc autoriser la mise en scène du cadavre mutilé de Phaéton-Coligny (contrairement à la tragédie d'actualité de Chantelouve), ainsi que l'évocation, même discrète, des violences extrêmes de la fin août 1572. Même dans un contexte ultra-catholique, la représentation directe d'une telle cruauté apparaît impossible : le détour mythique n'est pas ici utilisé pour susciter une lecture allégorisée du réel, mais pour permettre à un présent insoutenable et très problématique de trouver une forme de représentation ${ }^{19}$.

\section{Représenter la violence par la distance : The Massacre at Paris de Christopher Marlowe}

Les massacres de 1572 semblent ainsi proposer une double impossibilité aux dramaturges catholiques qui souhaitent les évoquer dans leurs œuvres : d'une

19 Pour cet événement si différent qu'est la Saint-Barthélemy, le théâtre n'est d'ailleurs pas le seul art à user de ce détour détourné. La fresque que Giorgio Vasari a consacrée à la nuit du 24 août, pour répondre à une commande du pape Grégoire XIII, et qui est encore aujourd'hui présentée dans la Sala Regia des palais du Vatican, montre ainsi le massacre de vieillards par des jeunes soldats romains vêtus à l'antique. La défénestration d'un homme au second plan du tableau, qui évoque très certainement la mort de Coligny (le tableau fut peint dans le courant de l'année 1573), est le seul élément qui permette d'identifier le massacre, mais, dans le même temps, le « détour » est, comme dans Phaéton, incomplet : seuls les vêtements des massacreurs renvoient à l'histoire de Rome. Au contraire, ceux des Massacres du triumvirat d'Antoine Caron (1566) peuvent certes faire allusion aux premiers conflits religieux, mais en représentant le plus fidèlement possible un événement historique ancien, qui fait entièrement sens pour le spectateur en dehors de toute lecture allégorique. 
part aucun récit antérieur (biblique, mythologique ou historique) ne leur paraît pleinement adéquat ou suffisant pour dire la spécificité de cette violence extrême, quand bien même celle-ci serait justifiée d'un point de vue politique et/ou religieux; d'autre part il ne leur apparaît pas possible de représenter sur la scène la totalité de cet événement comme un événement présent, sans être saisis d'horreur et/ou de honte face au témoignage de la cruauté sanglante de leur camp. À mesure que l'enthousiasme sacré des massacres s'est dissipé, la nuit de la Saint-Barthélemy devient une actualité impossible à mettre en scène : qu'on lui dénie son caractère événementiel et qu'on réduise ses acteurs au statut de témoins impuissants (Chantelouve), ou bien qu'on la renvoie dans un passé mythologique destiné à dire mais aussi à cacher la dimension horriblement présente du massacre des protestants (Bellaud).

En guise de conclusion, nous voudrions à présent souligner comment le passage du temps semble opérer de manière tout à fait inverse pour la seule pièce d'actualité réformée qui représente sur le théâtre les événements de 1572, près de vingt ans après les faits ${ }^{20}$ : The Massacre at Paris de Christopher Marlowe. Alors que le temps écoulé depuis l'événement rend sa représentation de plus en plus complexe pour des dramaturges catholiques confrontés à leur propre fureur meurtrière, il apparaît au contraire comme la condition de possibilité d'une mise en scène protestante de l'événement. Après la sidération traumatique, le silence scénique causé par la désorganisation, le choc et le deuil, le temps de la propagande semble avoir sonné dans le camp protestant. Ce n'est cependant pas sur les scènes genevoises que la Saint-Barthélemy va être mise en scène - et ce peut-être en raison de la méfiance envers les représentations sanglantes depuis la condamnation des mystères de la Passion par Luther et, plus généralement, du fait de l'interdiction officielle des représentations théâtrales dans la cité calviniste. Autre pays, autre mœurs, autre esthétique : c'est sur la scène élisabéthaine que les massacres du 24 août 1572 ainsi que l'assassinat du duc de Guise (1588) ou celui d'Henri III (1589) accèdent à une représentation doublement directe (puisqu'ils sont mis en scène sous les yeux des spectateurs, dans une pièce d'actualité), mais aussi doublement distante, temporellement et spatialement.

Si nous sommes certains que la pièce de Marlowe a été jouée plusieurs fois par la compagnie dirigée par Philip Henslowe, les Serviteurs du Lord amiral, et avec succès, le texte qui a été publié autour de 1593 sous le titre The Massacre at Paris : with the death of the Duke of Guise est profondément incomplet et semble avoir

20 Si l'année exacte de rédaction du Massacre at Paris demeure incertaine, les critiques s'accordent aujourd'hui sur la date approximative de 1592 pour sa première représentation. 
été recomposé de mémoire par certains acteurs de la compagnie. Les effets de brièveté et de ruptures que nous pouvons constater dans The Massacre at Paris tel que nous le connaissons, s'ils s'inscrivent dans une esthétique propre au théâtre élisabéthain, doivent être nuancés par la certitude que nous ne possédons qu'un squelette de la pièce, ce qui accentue la succession précipitée des actions scéniques, au détriment du développement des caractères des personnages, dont les tirades sont anormalement courtes. Ces précautions prises, nous ne pouvons que constater l'extrême différence dans le traitement scénique de la violence entre la pièce de Marlowe et celle de Chantelouve. The Massacre at Paris met en effet sous les yeux des spectateurs un empoisonnement, dix-sept meurtres dont un régicide, une mort suspecte (celle du roi Charles IX), ainsi que les sévices pratiqués sur le cadavre de l'amiral de Coligny. Un effet de massacre de masse est créé par l'alternance des scènes d'assassinats individuels (Coligny, Séroune, Ramus, les « instituteurs 》 de Condé et Navarre) et des scènes de tueries collectives, qui insistent sur le fait que la Saint-Barthélemy n'a pas seulement visé les chefs d'un éventuel complot mais aussi de nombreux protestants anonymes. La répétition des impératifs « Tue! », en français dans le texte, et des didascalies internes qui enjoignent à certains personnages de débarrasser la scène des cadavres pour permettre le déroulement de la scène (de massacre) suivante, cherche à susciter un effet d'horreur, de répulsion et de saturation, pour ainsi dire, chez le spectateur anglais protestant, qui ne peut que compatir avec les victimes huguenotes impuissantes, qu'elles soient de haut rang (Coligny), lettrées (Ramus, les « instituteurs ») ou issues du peuple (Séroune). La responsabilité du massacre n'est de sucroît pas seulement attribuée à Guise, à Catherine de Médicis et à Anjou ; les tueries sont aussi l'œuvre du peuple parisien, qui traite avec le plus grand mépris la dépouille de l'amiral.

Il s'agit pour Marlowe de dénoncer la Saint-Barthélemy par la mise en scène d'une violence extrême et itérative, qui semble ne pas avoir d'autre fin qu'elle-même et à laquelle il dénie toute valeur sacrée. Le duc de Guise, héros noir de la pièce, est ainsi le pendant du Coligny de la pièce de Chantelouve ${ }^{21}$ : ambitieux et athée, il se sert de la religion catholique et du soutien que lui offrent Catherine de Médicis, le pape et le roi d'Espagne pour servir son ascension personnelle. Henri III n'est pas épargné : bourreau masqué des protestants pendant les massacres, il est ensuite

21 R. Hillman a montré qu'il n'était pas impossible que Marlowe ait eu connaissance de la pièce de Chantelouve : voir Fr. de Chantelouve, The Tragedy of the Late Gaspard de Coligny, éd. et trad. R. Hillman, Ottawa, Dovehouse Editions, « Carleton Renaissance Plays in Translation », 2005, p. 22. 
un roi dominé par ses passions et ses mignons, qui se livre à un assassinat peu glorieux sur la personne du duc de Guise, pour mourir finalement sous les coups d'un moine jésuite. Les violences commises contre les protestants dans la première partie de la pièce semblent ainsi se retourner contre les bourreaux catholiques : à la mort de Coligny trahi par Charles IX répond la mort du duc de Guise trahi par Henri III, à la mort du pasteur Loreine celle du cardinal de Guise, à la mort par empoisonnement de la reine Jeanne de Navarre le couteau empoisonné de Jacques Clément. L'accession au trône d'Henri de Navarre à la fin de la pièce mettra-t-elle un terme à cette ronde sanglante ? Rien n'est moins sûr car Henri III, devenu subitement anti-catholique, demande à Navarre de le venger et à Épernon d'affuter son épée sur les os du pape Sixte V, et de tous les catholiques. En 1592, ce propos anti-catholique doit plaire dans une Angleterre victorieuse de la flotte espagnole en 1588, et plus particulièrement à la reine Élisabeth, qui tente de justifier auprès de ses sujets le soutien qu'elle apporte à Henri IV depuis 1589.

Si l'état du texte du Massacre at Paris doit nous inciter à la prudence dans l'analyse, la certitude que nous avons que la pièce mettait en scène de manière directe les tueries de la fin de l'été 1572 nous permet tout de même d'établir les conditions de possibilité de la représentation de cette actualité dans le théâtre protestant. Avant toute chose, la distance temporelle (20 ans), conjuguée à la distance spatiale (l'Angleterre est une île, même si les échanges entre les deux pays sont intenses, et les réfugiés huguenots privilégient Genève ou les Pays-Bas pour leur exil), semble permettre le dépassement du trauma initial. D'autre part, le point de vue est protestant et anti-catholique : une fois le choc passé, il est possible pour un dramaturge réformé de convoquer cet événement traumatique pour en faire un support de propagande pathétique et appeler les protestants à la vengeance armée contre les catholiques - alors que l'utilisation de la SaintBarthélemy à des fins de propagande semble extrêmement délicate pour un dramaturge catholique comme Chantelouve.

Enfin, la distance esthétique qui sépare le théâtre anglais du théâtre francophone semble être un élément déterminant : pour pouvoir mettre en scène cet événement sanglant, il n'est pas seulement nécessaire pour le dramaturge de pouvoir assumer (politiquement et affectivement) la force pathétique des émotions qu'il va susciter chez les spectateurs (pitié, horreur, compassion), il lui faut de plus avoir à sa disposition les catégories esthétiques et les conditions pratiques qui lui permettent d'en envisager la représentation. Ce n'est ainsi pas un hasard si c'est dans le «wooden $0^{22} 》$, cet espace polyvalent, ouvert, dans lequel

22 W. Shakespeare, Henry V, lère partie, Prologue. 
peuvent alterner scènes de foule et scènes intimes jouées très près de spectateurs familiers de la représentation de la violence sur la scène, que le massacre de la Saint-Barthélemy a trouvé un espace de représentation directe, dans une actualité certes élargie mais encore brûlante, à un moment où la succession au trône de France et l'avenir religieux du royaume ne sont pas encore résolus. Au contraire, pour les dramaturges français comme François de Chantelouve, l'influence de la tragédie humaniste antiquisante, qui ne refuse pas la mise en scène des actes sanglants, mais les utilise de manière beaucoup plus limitée que le théâtre élisabéthain en privilégiant plutôt le récit et en ne comportant pas de scènes de foule, s'allie au silence politique et au déni que les catholiques et la monarchie font peser sur les massacres, pour rendre impossible la représentation scénique directe de l'actualité sanglante que constitue la nuit de la Saint-Barthélemy.

Charlotte Bouteille-Meister Université Paris Ouest Nanterre La Défense 